\title{
An Analysis and Computation of Optimum Earth Geographical Coverage for Global Satellite Communications
}

\author{
O. C. Eke Vincent ${ }^{1 *}$, A. N. Nzeako ${ }^{2}$ \\ ${ }^{1}$ Department of Computer Science, Ebonyi State University, Abakaliki, Nigeria \\ ${ }^{2}$ Department of Electronic Engineering, University of Nigeria, Nsukka, Nigeria \\ Email: *veke39@yahoo.com
}

Received July 19, 2013; revised August 19, 2013; accepted August 26, 2013

Copyright (C 2013 O. C. Eke Vincent, A. N. Nzeako. This is an open access article distributed under the Creative Commons Attribution License, which permits unrestricted use, distribution, and reproduction in any medium, provided the original work is properly cited.

\begin{abstract}
This paper presents simple mathematical mobility models for configuration of Global interconnectivity with LEO satellite systems. The aim of this paper is to investigate on the performance measures of the satellite mobility models regarding optimum Global coverage arc length depending on the satellite locations relative to the four zones (quadrants) of the earth surface. A typical body of the satellite was positioned at a modified height of $780 \mathrm{Km}$ from the earth surface and revolving round the earth in a circle of radius, $7160 \mathrm{Km}$ was carefully studied and analytically parameterized enabling the generation of realistic instantaneous coverage arc lengths data. We compared the minimum required instantaneous arc lengths for the three mobility models that should cover the geographical coverage areas of the earth. The impact of the satellite movements relative to the earth locations was that the instantaneous coverage arc lengths were exponentially varying with time and continuously distributed within the four zones of the earth surface to provide continuous coverage around one polar orbit plane and assuming operations can continue down to an elevation angle of zero degree. The advantage of the derived mobility models is achieving almost $100 \%$ global coverage as a result of the dynamic behavior of the satellite playing an important role of providing instantaneous coverage arc lengths. This procedure also allows comparisons among different degrees of built-up zones of the earth surface as well as extra-polation to the different locations on the earth surface.
\end{abstract}

Keywords: Global Coverage; LEO Satellite; One Dimensional Model; Satellite Mobility Model; Central Angle; Instantaneous Coverage; Arc Length; Terrestrial Cellular Concept

\section{Introduction}

We are in the midst of a global information revolution brought about by the evolution of digital technology. Satellite communications systems are essential in establishing the global information infrastructure. The rapidly evolving information infrastructures will play a critical role in realizing the "global village" concept of the world [1]. In the last century, mobile personal communications have enjoyed an overwhelming success by using terrestrial cellular concept. Current terrestrial wireless network-based personal communications services (PCS) provide telecommunications services within a small geographical area [2]. To provide complete global coverage

"Corresponding author. to a diverse population, several mobile satellite systems (MSS), such as Iridium, Teledesic, odyssey and Intermediate circular orbit (ICO) satellites have been commissioned [3]. In addition to providing global coverage, MSS can be connected to existing terrestrial-based telecommunication systems to share traffics [2]. Also satellite systems represent new ambitious solutions in an attempt of interconnecting fixed networks (Internet and ATM) worldwide for supporting real-time communications [4].

Satellite communication services can be provided by Geostationary earth orbit (GEO), Medium earth orbit (MEO), or Low earth orbit (LEO) satellites. Because of its much short distance from the earth, lower power requirements and smaller mobility terminal (MT) size, LEO satellite system is a preferable choice [5]. 
The analysis of a satellite system, through simulation, comprises many issues [4]: some of these are the precise definition of the coverage area as the inter-satellite is moving, establishment of the inter-satellite links (if the system employs ISLs), the dynamic routing in the derived topology, the minimization of path switching, the establishment of the up-down link, the handoff conditions and finally the pattern analysis of the traffic originating from or terminating at the earth stations. If the coverage area of a place must be continuous, a GEO orbit can be selected or a constellation of Non Geostationary satellite orbit (NGSO) satellites can be designed to provide the necessary coverage overlap between successive satellites. Since a LEO satellite is not located at a geosynchronous orbit, it is mobile with respect to a fixed point on the earth and it is orbiting the earth with a high constant speed at a relatively low altitude [6], which results in a motion seen from a reference point over the earth. Most use circular orbits, since a constant altitude means that the satellite overhead can be used for network traffic throughout its orbit. In addition, successive satellites arranged in circular orbits showing the orbital plane can provide continuous coverage of a strip of ground beneath them, "street of coverage". Many proposed systems use street of coverage.

It has been observed that for a system designer to develop a LEO constellation which provides continuous global coverage, the following design considerations are required [6]: the length of coverage arc on the surface of the earth within the instantaneous coverage, the gain of the satellite antenna (if one beam is to illuminate this coverage), the number of satellites needed to complete a global system. However, the first requirement needed further investigation as the following constraints have also been identified [6]: orbital height of $750 \mathrm{Km}$ due to user terminal power, operating time between battery charges, and satellite launcher capabilities.

It is also very important to state that several mobility models have been proposed in the recent literatures which aim to describe the movements of the satellite foot prints on the earth's surface. In almost all the studies, one dimensional model was employed [7]. This is predicated upon the view that the rotation of the earth is negligibly compared to the satellites movement. This is a simple model to work with; however, it is only valid for services that have a short mean duration. In [8], the two dimensional mobility models have been proposed and employed [9] which took account of the earth's rotation. It has also been observed that the satellite to which a call will be handed over is not always the following one in the orbital plane. However, if a user is located in the over-lapping area, then it is likely due to the earth's rotation that he/she will be handed over to a satellite of the contiguous orbital plane.
In our study, we intend to exploit the concept of the one-dimensional mobility models in understanding the performance of the satellite mobility models with a view of determining the optimum global coverage for mobile communications services. Thus the idea of this paper is structured to present the conceptual one-dimensional satellite mobility models, and analytically in order to understand the performance in terms of instantaneous arc length, time and coverage parameters.

\section{Theoretical Procedure}

We first of all consider the conceptual satellite mobility models in conjunction with analytical and numerical solutions that will enable us to use satellite mobility model parameters to analyze the modifications needed for the reduction of the orbital period between any two satellites placed exactly over the reference earth point; and sufficient for the consideration of any possible combination of the traffic distributions and constellation patterns [6].

\subsection{Description of the Conceptual Satellite Mobility Models}

In this section, we consider the geometrical aspects of determining coverage requirements. In Figure 1 below, A spacecraft orbits at a distance, $r_{s}$ from the centre of the earth C. $r_{s}$ is the vector from the centre of the earth to the satellite. $r_{e}$ is the vector from the centre of the earth to the earth station; and $d$ is the vector from the earth station to the satellite. These three vectors lie in the same plane and form a triangle. The central angle $\theta$ measured between $r_{s}$ and $r_{e}$ is the angle between the earth station and the satellite and $\psi$ is the angle measured from $r_{e}$ to $d$. The angle $\alpha$ gives the angular distance between two neighboring satellites of the same orbit.

We assume that the spacecraft is a communication satellite and that it needs to be in contact with an earth station located at E. In this scheme, user mobility and earth rotation speeds are ignored based on the assumption of short call holding times of circuit switched voice traffic. However, multimedia traffic has longer connection holding times [1]. The surface of the earth is divided up into a grid like structure of orthogonal lines: latitude and longitude which describes the locations of the earth stations that must communicate with the satellites, while the coordinates to which an earth station antenna must be pointed to communicate with the satellite are called look angles expressed as azimuth $A_{z}$ and elevation $E_{l}$ (or $\phi)$ angles. The earth station antenna designers use the local horizontal plane and geographical compass points to define the azimuth angle thus giving the two look angles $\left(A_{z}, E_{l}\right)$ for the earth station antenna towards the satellite as shown in Figure 1 below. 


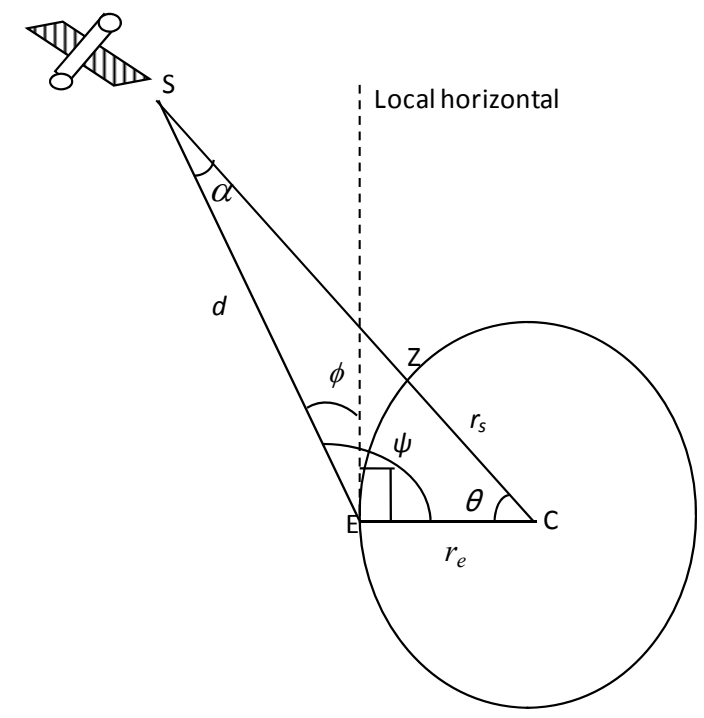

Figure 1. Geometry for calculating coverage area.

Using the sine rule to triangle SEC, we have that

$$
\left[r_{s / \sin (90+\theta)}\right]=[d / \sin \theta]
$$

Which yields

$$
\cos \phi=\left[r_{s} \sin \theta\right] / d
$$

All the three parameters, $\phi, \theta$, and $d$ in "Equation (1)" above have key inputs to the architecture of the satellite system [7]: The angle $\theta$ will yield the coverage area on the surface of the earth assuming the satellite has symmetrical coverage about nadir; the distance, $d$ will determine the free space path loss along the propagation path and will be a factor in the link budget design. The elevation angle $\phi$ will influence the G/T (Gain/system temperature) ratio of the antenna, the blockage probability and terrain and buildings near the antenna and the likely propagation impairments that will be encountered along the path to the satellite.

Most commercial satellite systems require that the earth stations operate above certain minimum elevation angles [6]. For example, INTELSAT $G$ band 6/4 $\mathrm{GHz}$ satellites operate above $50^{\circ}$. At $\mathrm{Ku}$ band (14/11 $\mathrm{GHz}, 14 / 12 \mathrm{GHz}$ ) INTELSAT satellites operate above a minimum elevation angle of $100^{\circ}$; while the Teledesic systems operate above $40^{\circ}$. Given a minimum elevation angle, $\phi$ and an orbital height, $h\left[=r_{s}-r_{e}\right]$, we can set up the geometry of Figure 1 above to develop a coverage area, assuming that the satellite has a symmetrical beam aimed at nadir. The central angle $\theta$, which will allow us to find the length of half of the arc under the coverage arc EZ can be found thus [6] using sine rule in Figure 1, we have:

$$
\sin \alpha / r_{e}=\sin \mathrm{SEC} / r_{s}
$$

$$
\begin{aligned}
\text { But angle } \mathrm{SEC} & =\psi=\phi+90^{\circ} \\
\therefore \alpha & =\sin ^{-1}\left[\sin \left(\phi+90^{\circ}\right) \frac{r_{e}}{r_{s}}\right]
\end{aligned}
$$$$
\text { Arc } \mathrm{EZ}=r_{e} \times \theta(\theta \text { in radians }) \mathrm{Km}
$$

The diameter of the instantaneous Coverage region is given by

$$
\text { 2Arc } \mathrm{EZ}=2\left[r_{e} \times \theta\right]
$$

And the coverage angle measured at the centre of the earth is given

$$
\theta=2 \times \alpha
$$

\subsection{Theoretical Analysis of the Satellite Mobility Models}

In the analysis of the motion of a satellite depicted in Figure 1 above, we consider a satellite body of mass $\mathrm{m}$ that is at a height, $h$ above the earth and is revolving round the earth in a circle of radius, $r_{s}$. The angular displacement, $\theta$ in radian, is given in terms of arc length $\ell$ it subtends on a circular radius, $r_{s}$ by [10] as

$$
\theta=l / r_{s}
$$

Or

$$
l=\theta r_{s}
$$

where $\ell$ is circumferential distance, a satellite body on the circle of rotation has moved (or would roll without slipping) if free to do so. This is Newton's law of circular motion. The fundamental laws of physics such as Newton's laws of motion, is given by [10] as:

$$
\theta=\theta_{0}+\omega_{0} t+1 / 2 a t^{2}
$$

These fundamental laws are usually based on empirical means, that is by observation and experiment that explains variations in physical properties and state of systems [11]. These fundamental laws define mechanisms of change and when combined with continuity laws for energy, mass or momentum differential equations result. Hence, we employ Equation (8) above to model the motion of a satellite in a circular orbit by substituting Equation (8) into Equation (7).

Thus,

$$
l_{1}=\theta r_{s}=r_{s}\left(\theta_{0}+\omega_{0} t+\frac{1}{2} a t^{2}\right)
$$

$$
\text { At } \begin{aligned}
t & =0, \quad \theta_{0}=0 \\
l_{1} & =r_{s}\left(\omega_{0} t+\frac{1}{2} a t^{2}\right)=r_{s} t\left(\omega_{0}+\frac{1}{2} a t\right) \\
& =r_{s} t\left(\frac{2 \omega_{0}}{a}+t\right)=r_{s} t\left(\frac{2 \omega_{0}}{a}+\frac{l}{v}\right)\left[\text { where } t=\frac{l}{v}\right] \\
& =r_{s} t\left(\frac{2 \omega_{0} v}{a}+l\right)
\end{aligned}
$$




$$
\therefore \frac{\mathrm{d} \ell}{\mathrm{d} t}=r_{s}\left(\frac{2 \omega_{0} v}{a}+l\right)
$$

The above Equation (10) relates the rate of change of the circumferential distance of a satellite body revolving the earth to the force acting on it. This is a differential (O.D.E) equation written in terms of the variables $(l, t)$ that we are interested on predicting. However, the exact solution of Equation (10) is difficult to be obtained by simple algebraic manipulation; rather we apply advanced calculus to solve it analytically.

\subsection{Analytical Modeling}

The analytical solution of Equation (10) above can be obtained thus: $\frac{\mathrm{d} l}{\mathrm{~d} t}=r_{s}\left(\frac{2 \omega_{0} v}{a}+l\right)$

Rearranging, we have $\mathrm{d} l-l=r_{s}\left(\frac{2 \omega_{0} v}{a}\right) \mathrm{d} t$

Then integrating both sides, we have

$$
\begin{gathered}
\int(\mathrm{d} \ell-l)=r_{s}\left(\frac{2 \omega_{0} v}{a}\right) \int \mathrm{d} t \\
\int\left(\frac{\mathrm{d} l}{l}-1\right)=r_{s}\left(\frac{2 \omega_{0} v}{a}\right) \int \mathrm{d} t \\
\log _{e} l=\left(\frac{2 r_{s} \omega_{0} v}{a}\right)^{t}+1 \\
\therefore l_{2}(t)=1+\mathrm{e}^{\left(\frac{2 r_{s} \omega_{0} v}{a}\right)^{t}}
\end{gathered}
$$

Equation (11) above is cast in a general mathematical model that expresses the essential features of the physical system that we are analyzing. Note that $l(t)$, is the dependent variable; $t$ is the independent variable, $r_{s}, \omega_{0}, v$ are the parameters, while $a$ is the driving (or forcing) function.

\subsection{Numerical Modeling}

Alternatively, we can reformate Equation (11) above using Euler's method [11] as follows:

The time rate of change of circumferential distance can be approximated by

$$
\frac{\mathrm{d} l}{\mathrm{~d} t} \cong \frac{\Delta l}{\Delta t}=\frac{l\left(t_{i+1}\right)-l(t)}{t_{i+1}-t_{i}}
$$

where $\Delta l$ and $\Delta t$ are differences in circumferential distance and time respectively computed over finite intervals and $l\left(t_{i}\right), l\left(t_{i+1}\right)$ are the circumferential distances at an initial time and some later time respectively.
Equation (12) is then called a finite divided difference approximation of the derivative at time, $t_{i}$.

Thus, substituting Equation (12) into Equation (11) gives

$$
\begin{gathered}
\frac{l\left(t_{i+1}\right)-l\left(t_{i}\right)}{t_{i+1}-t_{i}}=\frac{2 r_{s} \omega_{o} v}{a}+l\left(t_{i}\right) \\
l\left(t_{i+1}\right)-l\left(t_{i}\right)=\left(\frac{2 r_{s} \omega_{0} v}{a}+l\left(t_{i}\right)\right)\left(t_{i+1}-t_{i}\right) \\
\therefore l_{3}\left(t_{i+1}\right)=l\left(t_{i}\right)+\left[\frac{2 r_{s} \omega_{0} v}{a}+l\left(t_{i}\right)\right]\left[t_{i+1}-t_{i}\right] \\
=\left[2 l\left(t_{i}\right)+\frac{2 r_{s} \omega_{0} v}{a}\right]\left[t_{i+1}-t_{i}\right]
\end{gathered}
$$

Thus, the differential equation of Equation (11) has been transformed into an equation that can be used to determine the circumferential distance algebraically at $t_{i+1}$ using the slope and the previous values of $l$ and $t$ respectively. Hence, at any time along the way,

New Value $=$ Old Value + slope $\times$ Step Size

\subsection{Computation of the Satellite Mobility Model Parameters}

In this sub-section, we compute the satellite mobility model parameters by recalling that in [10]:
a) $\begin{aligned} r_{s} & =h+R_{e}=780 \mathrm{Km}+6380 \mathrm{Km}=7160 \mathrm{Km} \\ & =7,160,000 \mathrm{~m}\end{aligned}$
b) $V=R_{e} \sqrt{\frac{g}{r_{e}}}=638,000 \sqrt{\frac{9.8}{7,160,000}}=7464.1 \mathrm{~m} / \mathrm{s}$.
c) $\omega=\frac{V}{r_{s}}=\frac{7464.1}{7,160,000}=1.04 \times 10^{-3} \mathrm{~s}^{-1}$
d) $a=\frac{V^{2}}{r_{s}}=\frac{(7464.1)^{2}}{7,160,000}=7.78 \mathrm{~m} \cdot \mathrm{s}^{-2}$

e) The Period,

$$
\begin{aligned}
& T=\frac{2 \Pi r_{s}}{V}=\frac{2 \Pi \times 7,160,000}{7464.1}=6027.2 \mathrm{sec}=100.5 \mathrm{~min} \\
& \text { f) } l=2 \Pi r_{s}=2 \times 3.14 \times 7,160,000
\end{aligned}
$$

\subsection{Modifications}

The orbital altitude of the proposed system is taken equal to $780 \mathrm{Km}$. This modification is necessary for the reduction of the system period (The time needed for two satellites to be placed exactly over the reference earth point) to be approximately 100.5 minutes. This simulation period is sufficient for the consideration of any possible 
combination of traffic distribution and constellation pattern [10]. Hence, for our system, the length of the Global coverage is $l=44,964,800 \mathrm{~m}$ (or $44964.8 \mathrm{Km}$ ) and the orbital period is 100.5 minutes.

By inserting the numerical values of the calculated parameters in our derived equations, we have:

$$
\begin{aligned}
& l_{1}=r_{s}\left(\omega_{0} t+\frac{1}{2} a t^{2}\right) \\
& =7,160,000\left(1.04 \times 10^{-3} s^{-1} t+\frac{1}{2} \times 7.78 \frac{m}{s^{2}} \times t^{2}\right) \\
& =716,000\left(t+389 t^{2}\right) \\
& \quad l_{2}=l(t)=1+\mathrm{e}^{\left(\frac{2 r_{s} \omega_{0} V}{a}\right)^{t}} \\
& =1+\mathrm{e}^{\left(2 \times 7,160,000 \times 1.04 \times 10^{-3} \times 7464.1 / 7.78\right)^{t}} \\
& =1+\mathrm{e}^{1.4 \times 10^{7} t} \\
& l_{3}=l\left(t_{i+1}\right) \\
& =2 l\left(t_{i+1}\right)+\left[\frac{2 r_{s} \omega_{0} v}{a}\right] \times\left(t_{i+1}-t_{i}\right) \\
& =2 l\left(t_{i}\right)+\left[\frac{2 \times 7,160,000 \times 1.04 \times 10^{-3} \times 7464.1}{7.78}\right] \times\left[t_{i+1}-t_{i}\right] \\
& =2 l\left(t_{i}\right)+\left[1.4 \times 10^{7}\right] \times\left[t_{i+1}-t_{i}\right]
\end{aligned}
$$

\section{Results and Discussion}

Using the calculated system parameter values in Section2.4, we therefore insert these values in our derived equations of Equation (9), Equation (11) and Equation
(13) to get Equations (15), (16) and (17), respectively. A table of values for our $l_{1}, l_{2}, l_{3}$ as well as $\theta_{1}=l_{1} / r_{s}, \theta_{2}=l_{2} / r_{s}, \theta_{3}=l_{3} / r_{s} \quad$ values were computed and results tabulated as shown in Table 1 below.

Also values for our $l_{1}, l_{2}, l_{3}$ as well as $\theta_{1}=l_{1} / r_{s}$, $\theta_{2}=l_{2} / r_{s}, \theta_{3}=l_{3} / r_{s}$ values were computed using statistical analysis of time series and results tabulated as shown in Table 2 below.

The graph of Figure 2 below shows the series of cyclical variations of the instantaneous arc lengths of the global geographical coverage of a communications system. These series of cyclical variations occur at different points of time and are represented by quarterly time series in intervals of 3 minutes as shown Table 2 below. The moving averages for Equation (9) (or Equation (15)) given by series 1 (S1), Equation (11) (or Equation (16)) given by series 1 (S2), show a rise and fall in the time interval of 0 - 3 minutes. and 3 - 6 minutes., 6 - 9 minutes., 9 - 12 minutes, respectively while Equation (13) (or Equation (17)), shows a steadily increase in average moving from 0 - 12 minutes.

The best simulation results were obtained using Equation (13) (or Equation (17)) when compared with Equation (9) (or Equation (15)) and Equation (11) (or Equation (16)) respectively. The angular distance L3 ranges from $1.4 \times 10^{7}$ to $1.4 \times 10^{10}$ with moving averages from

\begin{tabular}{|c|c|c|c|c|c|c|c|}
\hline $\mathrm{S} / \mathrm{N}$ & $t(\mathrm{sec})$ & $l_{1}(\mathrm{~m})$ & $l_{2}(\mathrm{~m})$ & $l_{3}(\mathrm{~m})$ & $\theta_{1}=\left(\frac{l_{1}}{r_{s}}\right)^{0}$ & $\theta_{2}=\left(\frac{l_{2}}{r_{s}}\right)^{0}$ & $\theta_{3}=\left(\frac{l_{3}}{r_{s}}\right)^{0}$ \\
\hline 1 & 0 & 0 & 2 & 0 & 0 & 0 & 0 \\
\hline 2 & 1 & $2.8 \times 10^{9}$ & $1.28 \times 10^{6}$ & $1.4 \times 10^{7}$ & 7.8 & 0 & 165.6 \\
\hline 3 & 2 & $1.1 \times 10^{12}$ & $1.3 \times 10^{12}$ & $4.1 \times 10^{7}$ & 13.8 & 0 & 212.4 \\
\hline 4 & 3 & $4.2 \times 10^{14}$ & $1.5 \times 10^{19}$ & $9.6 \times 10^{7}$ & 64.4 & 0 & 151.2 \\
\hline 5 & 4 & $1.6 \times 10^{17}$ & $1.8 \times 10^{24}$ & $2.0 \times 10^{8}$ & 164.4 & 0 & 216.0 \\
\hline 6 & 5 & $6.4 \times 10^{19}$ & $2.0 \times 10^{30}$ & $4.3 \times 10^{8}$ & 151.3 & 0.2 & 277.2 \\
\hline 7 & 6 & $1.5 \times 10^{22}$ & $2.3 \times 10^{36}$ & $8.6 \times 10^{8}$ & 144.0 & 1.3 & 241.2 \\
\hline 8 & 7 & $9.6 \times 10^{24}$ & $2.6 \times 10^{42}$ & $1.7 \times 10^{9}$ & 52.2 & 9.2 & 7.2 \\
\hline 9 & 8 & $3.8 \times 10^{27}$ & $3.1 \times 10^{48}$ & $3.5 \times 10^{9}$ & 122.4 & 67.7 & 176.4 \\
\hline 10 & 9 & $1.5 \times 10^{30}$ & $3.6 \times 10^{54}$ & $7.0 \times 10^{9}$ & 90.0 & 136.8 & 313.2 \\
\hline 11 & 10 & $5.7 \times 10^{32}$ & $4.1 \times 10^{60}$ & $1.4 \times 10^{10}$ & 93.6 & 49.0 & 205.2 \\
\hline
\end{tabular}
0.000002 to 0.000005 with linear increase in length as shown in both Table 2 and Figure 2 respectively. It also has an angular coverage of $0^{\circ}$ to $313^{\circ}$ with $7.2^{\circ}$ being optimum (as shown in Table 1).

This translates into having an optimum of fifty satellites in an orbit. A value higher than this leads to larger gaps between the footprints of different satellites, so that connection is lost during the time a ground station is

Table 1. A Table of values of $l_{1}(\mathrm{~m}), l_{2}(\mathrm{~m}), l_{3}(\mathrm{~m})$ vs $t(\mathrm{sec})$ as well as $\theta_{1}, \theta_{2} \theta_{3}$. 
Table 2. A statistical table of moving point average values for Series of instantaneous arc lengths versus time series.

\begin{tabular}{cccccc}
\hline & \multirow{2}{*}{$\begin{array}{c}\text { Quarterly time series in intervals } \\
\text { of 3 mins }(t(\mathrm{~s}) .\end{array}$} & $\begin{array}{c}\text { Categories (CAT) of Quarterly } \\
\text { intervals of 3 mins }(t(\mathrm{~s}) \text { in }\end{array}$ & \multicolumn{2}{c}{$\begin{array}{c}\text { Moving Point average values for Series (S1, S2, S3) of } \\
\text { instantaneous arc lengths (L1, L2, L3) per millions: }\end{array}$} \\
\cline { 4 - 6 } & $0-3$ & 1 & L1 (or S1) & L2 (or S2) & L3 (or S3) \\
\hline 1 & $3-6$ & 2 & 0.0000043 & 0.0000024 & 0.000002 \\
3 & $6-9$ & 3 & 0.0000025 & 0.0000044 & 0.000002 \\
4 & $9-12$ & 4 & 0.0000035 & 0.0000018 & 0.000003 \\
\hline
\end{tabular}

LEGEND: Category means a set of point values of L (L1-L3) in quarterly intervals of time. Series mean a set of cyclical variations of the instantaneous arc length point values.

$\begin{array}{lc}\sim \text { Series } 1 & -\square \text { Series } 2 \\ -2 \text { per. Mov. Avg. (Series 1) }-2 \text { per. Mov. Avg. (Series 2) }-2 \text { per. Mov. Avg. (Series 3) }\end{array}$

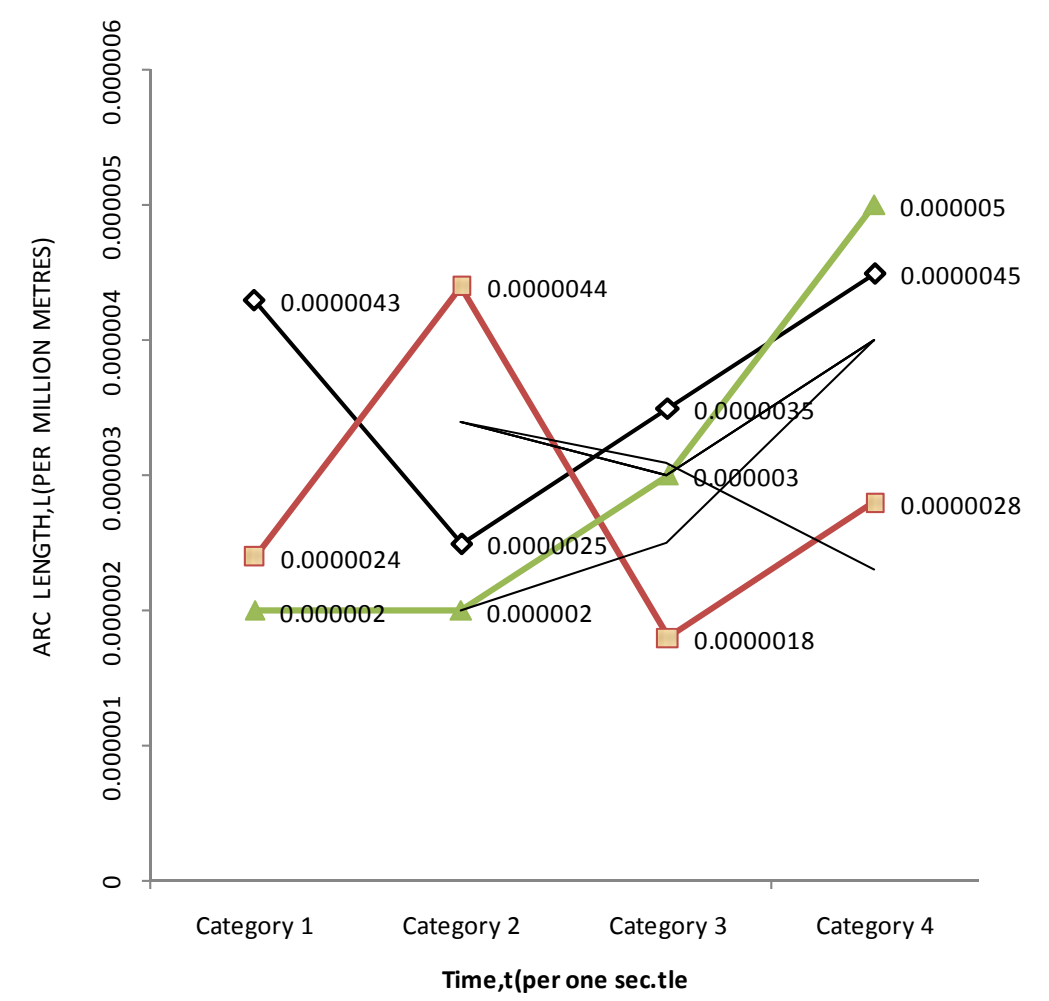

Figure 2. Graph of instantaneous arc length vs time.

inside such a gap. On the other hand, a low value has the effect that the period of time, in which both ground stations are in the footprints of the satellites, is very short which means frequent handover between adjacent satellites.

To an observer on the ground, the satellite appears to have infinite orbital period. It always stays in the same place in the sky [3]. Hence the instantaneous coverage arc is exponentially increasing with time as observed in Table 1 above using Equation (13) (or Equation (17)). In $[5,6]$, the authors found that the mean one way transmission time (OTT) cannot be accurately approximated by dividing OTTs in half i.e. the variation in the OTTs are often asymmetric. This is confirmed in our simulation work (see Table 1 and Figure 2 above). For instance, the series of moving average values for quarterly instantaneous arc length point values for lengths L1, L2 and L3 are $\mathrm{S} 1=(0.0000043,0.0000025,0.0000035$ and $0.0000045) ; \mathrm{S} 2=(0.0000024,0.0000044,0.0000018$, and 0.0000028$) ; \mathrm{S} 3=(0.000002,0.000002,0.000003$ and 0.000005$)$ respectively. Hence, the three minutes interval of moving averages is very effective in this case. The general trend of 2 per moving average per series was also illustrated in the graph Figure 2. In our Table 1, it is also observed that our computed coverage angle values for angular distances $l_{1}, l_{2}, l_{3}$, for various values of time 
period ( 0 - 10 seconds) shows that coverage angles $\left(\theta_{1}, \theta_{2}, \theta_{3}\right)$ fall within various quadrants of the Globe. For instance, $\theta_{1}$ covers the first and second quadrants of the range $7.8^{\circ}$ to $164.4^{\circ}, \theta_{2}$ covers the first and second quadrants of the earth in the range of $0^{\circ}-136.8^{\circ}$ while $\theta_{3}$ covers the first, second, third and even the fourth quadrants of the of the earth in the range of $7.2^{\circ}$ $313^{\circ}$ with Equation (13) or (17). This shows the best coverage of the Globe.

\section{Conclusions and Further Research}

An in-depth analysis and computation of optimum earth geographical coverage for Global satellite communications have been presented in the paper. A successful development of the satellite mobility models is presented and the performance of the satellite mobility models is evaluated in terms of the instantaneous arc length, time, and coverage angle parameters through mathematical simulations.

A comparison of the three developed satellite models has been made for developing earth geographical coverage for satellite Global communications. We believe that an optimization technique can approximate this asymmetric variation very effectively in the instantaneous arc lengths and this is exactly an interesting point for further investigation. System performances such as global coverage decisions, low handover rate requirements, acceptable transmission delay etc. need further research.

\section{REFERENCES}

[1] P. Chitre and F. Yegenoglu, "Next Generation Satellite Networks: Architectures and Implementations," IEEE Communications Magazine, Vol. 37, No. 3, 1999, pp. 3036.

[2] P. K. Chowdhury, M. Atiquzzama, et al., "Handover
Schemes in Space Networks: Classification and Performance Comparison," 2nd IEEE International Conference on Space Mission Challenges for Information Technology, Pasadena, 17-20 July 2006, pp. 8-108.

[3] I. F. Akyildiz, H. Uzunalioglu and M. D. Bender, "Handover Management in Low Earth Orbit (LEO) Satellite,” Mobile Networks and Applications, Vol. 4, No. 4, 1999, pp. 301-310.

[4] E. Papatron, et al., "Performance Evaluation of LEO Satellite Constellations with Inter-Satellite Links under SelfSimilar and Passion Traffic,” John Wiley \& Sons, Ltd., Hoboken, 1999.

[5] S. Karapantazis, et al., "On Call Admission Control and Handover Management in Multimedia LEO Satellite Systems," Proceedings of the 23rd AIAA International Communications Satellite System Conference, Rome, 25-28 September 2005.

http//newton.ee.auth.gr/pavliodou/papers/co56.pdf

[6] T. Pratt, et al., "Satellite Communications," 2nd Edition, John Wiley, Ltd., New York, 2003, pp. 52-432.

[7] S. Karapantazis, et al., "On Bandwidth and Inter-Satellite Handover Management in Multimedia LEO Satellite Systems," 23rd AIAA International Communications Satellite Systems Conference (ICSSC 2005), Rome, 25-28 September 2005. http//newton.ee.auth.gr/pavliodou/papers/co59.pdf

[8] V. Paxson, “End-to-End Internet Packet Dynamics," IEEE/ACM Transactions on Networking, Vol. 7, No. 3, 1999, pp. 277-292.

[9] K. Claffy, G. C. Polyzos and H.-W. Braun, "Measurement Considerations for Assessing Unidirectional Latencies,” Vol. 4, No. 3, 1993, pp. 121-132.

[10] F. W. Sears, M. W. Zemasnsky and H. D. Young, "University Physics," 6th Edition, Addison Wesley, New York, 1981, pp. 181-182.

[11] S. C. chapra and R. P. Canale, "Numerical Methods for Engineers,” 5th Edition, McGraw Hill Inc., New York, 2006, p. 17. 